\title{
Editorial comment: bladder preservation as a treatment strategy in muscle-invasive bladder cancer
}

\author{
Khaled Ajib, Girish S. Kulkarni \\ Division of Urology, Departments of Surgery and Surgical Oncology, Princess Margaret Cancer Center, University Health Network, University of \\ Toronto, Toronto, Canada \\ Correspondence to: Dr. Girish S. Kulkarni. Division of Urology, University Health Network Associate Professor, Urologic Oncology, University of \\ Toronto, Toronto, Canada. Email: Girish.kulkarni@uhn.ca, \\ Provenance: This is an Invited Editorial commissioned by Section Editor Xiao Li (Department of Urology, Jiangsu Cancer Hospital \& Jiangsu \\ Institute of Cancer Research \& Nanjing Medical University Affiliated Cancer Hospital, Nanjing, China). \\ Comment on: Audenet F, Waingankar N, Ferket BS, et al. Effectiveness of Transurethral Resection plus Systemic Chemotherapy as Definitive \\ Treatment for Muscle Invasive Bladder Cancer in Population Level Data. J Urol 2018;200:996-1004.
}

Submitted Sep 21, 2018. Accepted for publication Sep 30, 2018.

doi: $10.21037 /$ tau. 2018.10 .01

View this article at: http://dx.doi.org/10.21037/tau.2018.10.01

Bladder cancer is the eleventh most commonly diagnosed cancer worldwide and the fourth most common cancer in men in the United States $(1,2)$. In 2017, an estimated 17,000 deaths occurred from bladder cancer (2). $20 \%$ to $30 \%$ of patients diagnosed with non-muscle invasive bladder cancer progress to muscle invasive bladder cancer (MIBC), and $25 \%$ of MIBC are de novo presentations (3). Radical cystectomy is still considered the mainstay of treatment for localized, non-metastatic MIBC. However, the oncological outcomes are not perfect with observed 5 -year overall survival rates of $50 \%$ in all centers undergoing radical cystectomy (4). Moreover, this procedure is a major operation associated with an increased risk of morbidity and impaired quality of life (5), for which many elderly or infirmed patients are considered unfit. Other treatment modalities that include combinations of chemotherapy and/ or radiotherapy have been studied as alternatives to radical cystectomy (6). Bladder preservation methods promise a better quality of life without compromising the oncological outcome, but additional studies are required to substantiate this claim and to determine the optimal alternative. Furthermore, as most bladder-sparing regimens come with less toxicity than cystectomy, they may serve as appropriate alternatives for patients medically unfit for cystectomy.

In their article entitled "Effectiveness of transurethral resection plus systemic chemotherapy as a definitive treatment for muscle-invasive bladder cancer in population level data", published in the Fournal of Urology [2018], Audenet and colleagues studied the effectiveness of TURBT and chemotherapy alone in the management of MIBC (7). This was the first large-scale observational study to examine TUR plus chemotherapy as a definitive treatment of MIBC. A cohort of 1,538 patients (cT2-4N0M0), treated at several US medical centers, was identified using the National Cancer Database (NCDB). As an exploratory comparison, a localized (cT2-4NOM0) MIBC cystectomy cohort from the same time period [2004-2015] was selected from the same database (7). Patients in the cystectomy group may or may not have received perioperative chemotherapy. The TUR plus chemotherapy group included mainly elderly, uninsured, African American and surgically unfit patients (7).

The authors reported a higher postoperative mortality rate at 30 and 60 days in the cystectomy group compared to the TUR plus chemotherapy group ( $2.6 \%$ vs. $0.2 \%$ and $6.5 \%$ vs. $3.8 \%$, respectively) (7). These observations are not surprising given that radical cystectomy, a complex surgical procedure, is known to come with a not insignificant postoperative mortality rate (8). On the other hand, the overall survival was significantly lower in the TUR plus chemotherapy group (32.9\%) with a median survival of 23.9 months compared to 48.1 months in the RC group. Ultimately, the authors concluded that TUR plus chemotherapy provides an achievable longterm overall survival benefit, and indirectly supports the 
notion that some MIBC patients experiencing a complete clinical response to neoadjuvant may one day avoid radical cystectomy (7).

The authors should be congratulated for this important work and for addressing a question that has previously only been studied in small patient cohorts and/or single institution series. Their study is the largest reported to date and provides a real-world glance at the possibility of bladder preserving strategies that take advantage of complete responses post-neoadjuvant or induction chemotherapy. The data presented support the proof of principle that select patients treated with neoadjuvant chemotherapy and TUR alone can achieve long-term survival. A provocative interpretation of the results suggests radical cystectomy may be avoided in $1 / 3$ of patients, but the authors acknowledge the need for new trial initiatives to definitively prove this supposition.

Although this was the first large-scale observational study examining the role of TUR plus chemotherapy alone as treatment for MIBC, several smaller, single institution studies have been reported. In Miyata et al., the 5-year overall survival in the bladder preservation cohort of TUR plus systemic chemotherapy was higher (42\%) than in this series, perhaps arising from a difference in patient profile (9). Another cohort of 127 patients treated with intra-arterial Cisplatin regimens and TUR only achieved a $71.7 \%$ complete response rate with a 5 -year overall survival of $50.2 \%$ (10), pointing to the importance of understanding the mode and type of chemotherapy administered. Sternberg et al. studied the difference between TURBT, radical cystectomy, or partial cystectomy in patients who had neoadjuvant chemotherapy. They reported an overall survival of $60 \%$ in the 52 patients who had neoadjuvant chemotherapy + TUR (median follow-up 56 months) (11). Clearly, tumor pathological downstaging with neoadjuvant chemotherapy can have a major effect on survival outcomes. Numerous series have corroborated a pT0 rate of $25-30 \%$ with neoadjuvant chemotherapy (12-14). These studies indirectly suggest that radical cystectomy may potentially be overtreatment in patients with a $\mathrm{pT} 0$ response.

Our group recently studied an alternative method of bladder preservation with TUR plus combination chemoradiotherapy (TMT, trimodal therapy) (6). In this study, we demonstrated that, in carefully selected patients, both disease-specific and overall survival outcomes for TMT and radical cystectomy are comparable (6). Others, however, have used population-based datasets for similar comparison and found improved survival outcomes with radical cystectomy. For example, Zhong et al. utilized the National Cancer Database to analyze the outcomes of bladder preservation with chemoradiation versus radical cystectomy. The overall survival in the TMT group was 2.6 years compared to 3.6 years in the RC cohort (15). In their series, bladder preservation patients were older and more comorbid. Seisen et al., found identical results using the same database (16). Similarly, two independent studies by Bekelman et al. and Williams et al. showed a decrease in survival with TMT compared to radical cystectomy using data from the Surveillance, Epidemiology, and End ResultsMedicare linked database $(17,18)$. A plausible explanation for the discrepant results between our institutional study and those arising from population-based datasets is simply patient selection and confounding by indication. Specifically, the reasons for bladder preservation in population-based datasets are not available and may reflect higher age and a worse comorbidity profile.

Likewise, patient selection probably played a similar role in the study of Audenet $e t a l$. as there was no explicit criteria available stating why certain patients had TUR plus chemotherapy versus the gold standard radical cystectomy. The TUR plus chemotherapy group included mainly elderly, poor, uninsured, and surgically ineligible patients with high Charlson comorbidity scores (7). The risk profile of these patients suggests that TUR plus chemotherapy was given as a "treatment of last resort". If the intention was to cure rather than to palliate without cystectomy, one has to wonder why concomitant radiation was not delivered? Further supporting this notion is the lack of radical or repeat TUR to clear residual cancer in the TUR plus chemotherapy group. The Massachusetts General Hospital group has established that complete resection with repeat TUR improves complete response and TMT success rates (19). In Audenet's study, the number of TUR procedures was not provided nor were details given regarding the chemotherapy regimens utilized (7). These limitations, plus the inherent biases associated with retrospective studies including patient selection, make the results of the study difficult to interpret and likely explain the low overall survival rates reported for these patients. The lack of disease-specific survival in the NCDB further compounds these short-comings as competing risks could not be assessed. Many of the issues discussed above were also addressed in the 3 short editorial comments that accompanied the original manuscript. 
Despite these limitations, the real-world data described in this study highlight the importance of evaluating bladder preservation strategies in patients with MIBC. This study provides outcomes data for an alternative treatment in MIBC, which is value added. Finally, these data can also serve as a backbone for patient counseling prior to treatment.

With an overall survival of $32.9 \%$, this observational research paper showed that long-term survival can be achieved in MIBC patients treated with chemotherapy and TUR only. Although the success rate was quite low for localized, non-metastatic disease, this study supports further research assessing bladder preservation strategies, particularly those that build off of complete response rates with neoadjuvant chemotherapy. Further research is needed to identify the ideal treatment regimen and to identify predictive biomarkers of complete response to neoadjuvant chemotherapy and subsequent bladder preservation.

\section{Acknowledgements}

None.

\section{Footnote}

Conflicts of Interest: The authors have no conflicts of interest to declare.

\section{References}

1. Ferlay J, Soerjomataram I, Dikshit R, et al. Cancer incidence and mortality worldwide: sources, methods and major patterns in GLOBOCAN 2012. Int J Cancer 2015;136:E359-86.

2. American Cancer Society. Cancer Facts \& Figures 2017. Atlanta, GA: American Cancer Society; 2017.

3. Prout GR, Marshall VF. The prognosis with untreated bladder tumors. Cancer 1956;9:551-8.

4. Arcangeli G, Strigari L, Arcangeli S. Radical cystectomy versus organ-sparing trimodality treatment in muscleinvasive bladder cancer: A systematic review of clinical trials. Crit Rev Oncol Hematol 2015;95:387-96.

5. Cookson MS. The surgical management of muscle invasive bladder cancer: a contemporary review. Semin Radiat Oncol 2005;15:10-8.

6. Kulkarni GS, Hermanns T, Wei Y, et al. Propensity Score Analysis of Radical Cystectomy Versus Bladder-Sparing
Trimodal Therapy in the Setting of a Multidisciplinary Bladder Cancer Clinic. J Clin Oncol 2017;35:2299-305.

7. Audenet F, Waingankar N, Ferket BS, et al. Effectiveness of Transurethral Resection plus Systemic Chemotherapy as Definitive Treatment for Muscle Invasive Bladder Cancer in Population Level Data. J Urol 2018;200:996-1004.

8. Schiavina R, Borghesi M, Guidi M, et al. Perioperative complications and mortality after radical cystectomy when using a standardized reporting methodology. Clin Genitourin Cancer 2013;11:189-97.

9. Miyata Y, Nomata K, Ohba K, et al. Efficacy and safety of systemic chemotherapy and intra-arterial chemotherapy with/without radiotherapy for bladder preservation or as neo-adjuvant therapy in patients with muscle-invasive bladder cancer: a single-centre study of 163 patients. Eur J Surg Oncol 2015;41:361-7.

10. Han B, Liang S, Jing Y, et al. Organ preservation for muscle-invasive bladder cancer by preoperative intraarterial chemotherapy and transurethral resection. Med Oncol 2014;31:912.

11. Sternberg CN, Pansadoro V, Calabrò F, et al. Can patient selection for bladder preservation be based on response to chemotherapy? Cancer 2003;97:1644-52.

12. Rosenblatt R, Sherif A, Rintala E, et al. Pathologic downstaging is a surrogate marker for efficacy and increased survival following neoadjuvant chemotherapy and radical cystectomy for muscle-invasive urothelial bladder cancer. Eur Urol 2012;61:1229-38.

13. Meeks JJ, Taylor JM, Matsushita K, et al. Pathological response to neoadjuvant chemotherapy for muscle-invasive micropapillary bladder cancer. BJU Int 2013;111:E325-30.

14. Zargar H, Zargar-Shoshtari K, Lotan Y, et al. Final Pathological Stage after Neoadjuvant Chemotherapy and Radical Cystectomy for Bladder Cancer-Does pT0 Predict Better Survival than p'Ta/Tis/T1? J Urol 2016;195:886-93.

15. Zhong J, Switchenko J, Jegadeesh NK, et al. Comparison of Outcomes in Patients With Muscle-invasive Bladder Cancer Treated With Radical Cystectomy Versus Bladder Preservation. Am J Clin Oncol 2018. [Epub ahead of print].

16. Seisen T, Sun M, Lipsitz SR, et al. Comparative Effectiveness of Trimodal Therapy Versus Radical Cystectomy for Localized Muscle-invasive Urothelial Carcinoma of the Bladder. Eur Urol 2017;72:483-7.

17. Bekelman JE, Handorf EA, Guzzo T, et al. Radical cystectomy versus bladder-preserving therapy for muscleinvasive urothelial carcinoma: examining confounding and 
misclassification biasin cancer observational comparative effectiveness research. Value Health 2013;16:610-8.

18. Williams SB, Shan Y, Jazzar U, et al. Comparing Survival Outcomes and Costs Associated With Radical Cystectomy and Trimodal Therapy for Older Adults With Muscle-

Cite this article as: Ajib K, Kulkarni GS. Editorial comment: bladder preservation as a treatment strategy in muscle-invasive bladder cancer. Transl Androl Urol 2018;7(Suppl 6):S723-S726. doi: 10.21037/tau.2018.10.01
Invasive Bladder Cancer. JAMA Surg 2018;153:881-9.

19. Premo C, Apolo AB, Agarwal PK, et al. Trimodality therapy in bladder cancer: who, what, and when? Urol Clin North Am 2015;42:169-80, vii. 